University of Nebraska - Lincoln

DigitalCommons@University of Nebraska - Lincoln

Publications, Agencies and Staff of the U.S.

Department of Commerce

U.S. Department of Commerce

2007

Chronic Exposure to Polynuclear Aromatic Hydrocarbons in Natal

Habitats Leads to Decreased Equilibrium Size, Growth, and

Stability of Pink Salmon Populations

Ron A. Heintz

National Oceanic and Atmospheric Administration, ron.heintz@noaa.gov

Follow this and additional works at: https://digitalcommons.unl.edu/usdeptcommercepub

Part of the Environmental Sciences Commons

Heintz, Ron A., "Chronic Exposure to Polynuclear Aromatic Hydrocarbons in Natal Habitats Leads to Decreased Equilibrium Size, Growth, and Stability of Pink Salmon Populations" (2007). Publications, Agencies and Staff of the U.S. Department of Commerce. 280.

https://digitalcommons.unl.edu/usdeptcommercepub/280

This Article is brought to you for free and open access by the U.S. Department of Commerce at DigitalCommons@University of Nebraska - Lincoln. It has been accepted for inclusion in Publications, Agencies and Staff of the U.S. Department of Commerce by an authorized administrator of DigitalCommons@University of Nebraska - Lincoln. 


\title{
Chronic Exposure to Polynuclear Aromatic Hydrocarbons in Natal Habitats Leads to Decreased Equilibrium Size, Growth, and Stability of Pink Salmon Populations
}

\author{
Ron A Heintz* \\ Alaska Fisheries Science Center, National Marine Fisheries Service, National Oceanic and Atmospheric Administration, Auke Bay \\ Laboratory, 11305 Glacier Hwy, Juneau, Alaska 99801, USA
}

(Received 2 May 2006; Accepted 6 December 2006)

This article is a U.S. government work, and is not subject to copyright in the United States.

\begin{abstract}
The immediate and delayed effects of embryonic exposure to low levels of polynuclear aromatic hydrocarbons (PAHs) have been shown to reduce survival to maturity by $50 \%$ in exposed pink salmon populations. This suggests that chronically exposed populations could be extirpated over relatively few generations, but the effect of density dependence on extirpation rate is unknown. This study examines the interaction of PAH effects and randomly varying density dependence on a simulated population. The simulation derives from 70 years of observations made on a single pink salmon population and toxicity studies involving a hatchery population in the same watershed. Results from simulations involving exposure of $100 \%$ of the population to effects consistent with an aqueous PAH concentration of $18 \mathrm{~nL} / \mathrm{L}$ indicate an $80 \%$ decrease in population productivity and an $11 \%$ probability of extinction after 35 generations. In contrast, population growth rate declined by only $5 \%$. Further decreases in survival relative to that of observed PAH effects rapidly increase the probability of extinction. Data from these simulations demonstrate that, at low levels of exposure, density dependence can compensate for reduced population size and buffer the population against extinction. However, if equilibrium size is depressed sufficiently, random environmental variation overcomes the buffering effect of density dependence and extinction probability increases. These data demonstrate that extinction probability and population size are more sensitive measures of population effects than growth rate for wild populations regulated by density dependence.
\end{abstract}

Keywords: Polynuclear aromatic hydrocarbons Population Density dependence Salmon Toxicity

\section{INTRODUCTION}

Embryonic exposure to polynuclear aromatic hydrocarbons (PAHs) causes a variety of effects on individuals, but the cumulative impact of those effects on chronically exposed populations is not known. Numerous laboratory studies have highlighted the toxicity of $\mathrm{nL} / \mathrm{L}$ exposures to developing fish embryos, suggesting the potential for decreases in population size (Marty et al. 1997; Carls et al. 1999; Heintz et al. 1999; White et al. 1999; Coulliard 2002; Colavecchia et al. 2004). Furthermore, the survivors of these exposures can display sublethal effects that ultimately interact with other environmental stressors, further reducing the survival of the exposed population (Heintz et al. 2000). Also, high molecular weight PAHs have been shown to reduce the reproductive output of exposed fish (White et al. 1999). These effects on survivorship and fecundity suggest chronic exposure should produce effects on population size and growth, but environmental variation likely inhibits their detection (Pella and Myren 1974). These observations are troubling because our dependence on fossil fuels means PAHs unavoidably are released into aquatic habitats. In particular, estuaries, the primary natal habitat for many marine species, are sinks for these compounds (Kennish 2002). Consequently, it is important to understand how chronic contamination of natal habitats with low levels of PAHs influences the productivity, growth, and stability of aquatic populations.

Documenting population effects following exposure to PAHs at levels known to injure individuals has proven

To whom correspondence may be addressed: ron.heintz@noaa.gov difficult. Documented effects of contaminants on aquatic populations include reduced size (Geiger et al. 1996), altered age structure (Baumann et al. 1990), and extinction (Cook et al. 2003), but these are associated with catastrophic exposures. Demonstration of effects of chronic exposure to low levels of contaminants on natural populations is obscured by random variation in population size and a limited availability of demographic data (Landahl and Johnson 1993), yet these are precisely the sort of exposures typically encountered. Several attempts to understand how contaminants influence the dynamics of invertebrate populations have been reviewed by Forbes et al. (2001). These studies involved populations maintained in laboratories and their results indicate that the severity of contaminant effects on population growth rate depend on the types of individual effects and the compensatory ability of density dependence. Grant (1998) has argued that density-dependence buffers chronically exposed populations from extinction so that growth rates remain constant, but the equilibrium population size declines.

Model systems involving vertebrates generally are limited to combining published life history values with toxicity endpoints because of the expense and complexity involved in controlling the interactions between populations and their natural environments. To this end, Miller and Ankley (2004) combined the results of toxicity tests with published life history data for fathead minnows (Pimphales promelas) to demonstrate reduced equilibrium population size resulting from endocrine disruption. Similarly, Spromberg and Meador (2005) described a series of life table response experiments using theorized toxicity endpoints and published life history 
data collected from Chinook salmon (Oncorhynchus tshawytscha). Their theorized endpoints relied on the assumption that previously reported sublethal effects ultimately translate to reductions in survival. These effects have been demonstrated by Heintz et al. (2000), who reported delayed mortality among pink salmon (O. gorbuscha) that survived embryonic exposure to PAHs.

Pink salmon offer a particularly good vertebrate model for evaluating PAH impacts on aquatic populations. Pink salmon have a simple life history, easily are cultured, demonstrate a high degree of homing fidelity, and have well-described demographics. More importantly, the immediate and delayed effects following embryonic exposure have been quantified. Heintz et al. (1999) reported that aqueous concentrations as low as $1.0 \mathrm{~nL} / \mathrm{L}$ significantly can reduce survival during incubation. In addition, survivors of exposures of $5.4 \mathrm{~nL} / \mathrm{L}$ demonstrated significantly lower marine survival than unexposed controls (Heintz et al. 2000). Survivors of exposure had reduced growth rates, which were implicated as the cause for the observed reductions in survival. Survival reductions in each of the pink salmon life history stages combine to reduce the number of mature adults by about $50 \%$ in populations experiencing $15 \mathrm{~nL} / \mathrm{L}$ exposures (Heintz et al. 2000). Although survival estimates during incubation derive from cultured populations, the estimates describing delayed effects were made from cohorts released into the wild and recovered at maturity. Consequently, these estimates account for interactions between sublethal physiological impacts and the natural environment. Combining these data with demographic data provides a unique opportunity to examine population effects of PAH contamination in natal habitats.

The goal of this paper is to understand how chronic contamination of natal habitats with low levels of PAHs influences the equilibrium size, intrinsic growth rate, and stability of pink salmon populations. Equilibrium size is measured as the median number of returning adults, intrinsic growth as the median number of returns per spawner, and stability as the probability of extinction over 35 generations. These parameters are derived from simulating pink salmon population dynamics, using data collected from a population that has been monitored almost continuously since 1934. This population resides in the same watershed as the hatchery where the pink salmon toxicity studies took place. The simulation incorporates density-dependent functions for survival and random variation. In particular, the effect of increasing the extent of contamination in natal habitats is examined along with the impacts of decreasing survival.

\section{METHODS AND RESULTS}

Understanding the effects of chronic exposure to PAH on pink salmon requires development of a model pink salmon population and then manipulation of the model to identify the effects of exposure. Therefore there are 2 main sections to this report. The lst section motivates the model, describes how it was parameterized, and then outlines a series of tests performed to evaluate the model's reliability. The 2nd section extends the basic model by incorporating the effects of PAH exposure. Afterwards, the extended model is manipulated by introducing different exposure scenarios. These scenarios include the effects of increasing the proportion of the population exposed to the PAHs and reducing survival for individuals exposed to PAHs.

\section{Basic model}

Pink salmon are semelparous with an obligate 2-y life history. Eggs deposited in the gravel in fall incubate for approximately 7 months when fully formed fry emerge and immediately emigrate seaward. Many pink salmon streams harbor 2 distinct nonoverlapping populations, one that reproduces in odd-numbered years and the other that reproduces in even-numbered years. In a given stock, these 2 populations are genetically and behaviorally distinct and are referred to as the odd-year and even-year lines, respectively. Let $y_{t+2}$ equal the number of adults returning to a given stream. This number depends on the previous number of spawners $\left(y_{t}\right)$ by

$$
y_{t+2}=y_{t} s_{t}
$$

where the random variable $s_{t}$ represents the number of returns per spawner. The random variable $s_{t}$ incorporates the fecundity and survival of the progeny produced by $y_{t}$. Therefore, $s_{t}$ can be expressed as the product of fecundity per spawner and subsequent survival of the offspring as they transit all the developmental stages. Thus,

$$
s_{t}=f_{t} \times s f w_{t} \times s s w_{t}
$$

where $f_{t}$ is the individual fecundity from brood $t, s f w_{t}$ is the subsequent freshwater survival, and $s s w_{t}$ is saltwater survival for brood $t$. It is important to note that $f_{t}$ represents the entire fecundity of an individual, and $s f w_{t}$ accounts for the survival of only those offspring that were deposited successfully into the gravel. Consequently, $f_{t}$ must be adjusted by $p_{t}$, the proportion of eggs successfully deposited into the gravel making Equation 1,

$$
y_{t+2}=y_{t}\left(p_{t} \times f_{t} \times s f w_{t} \times s s w_{t}\right)
$$

Parameterization of the model requires estimates of these values and the respective oil effects.

Parameterization of basic model-Simulation of a pink salmon run requires estimates of the random variables $f_{t}$, $p_{t}$, $s f w_{t}$, and $s s w_{t}$ in order to derive an estimate $y_{t+2}$ from an arbitrarily selected value of $y_{t}$ (Eqn. 3). The Sashin Creek weir, located in southeastern Alaska, has been in operation almost continuously for nearly $70 \mathrm{y}$, providing counts of spawners from odd- and even-year lines for a period spanning 35 generations (Bradshaw and Heintz 2005). In addition, the number of emigrating fry has been monitored for a continuous 40-y period. For a 20-y subset of this period, the number of eggs deposited in the gravel also was monitored. These data provide opportunities for parameterizing density-dependent functions relating the fecundity of females to the number of eggs deposited in the stream, the survival during incubation of those eggs, and the marine survival of the resulting fry.

Estimates of the product $\left(y_{t} \times p_{t} \times f_{t}\right)$ from Equation 3 reflect the total number of eggs delivered to the stream by returning females that actually are deposited in the streambed. The number of eggs delivered to the stream is a product of random variables describing fecundity and the proportion of females in the run. The number deposited is a density-dependent function of the number delivered (McNeil 1964). To estimate the total number of eggs delivered to the stream by returning females, it is necessary to know the proportion of $y_{t}$ that is actually female and the average fecundities of individual females. Between 1940 and 1980, an average $49.2 \%$ of the returns were female, with a standard deviation of $4.7 \%$. The average fecundity of females during 
this period was $1,915 \pm 174$ eggs. The total number of eggs delivered to the stream in year $t\left(P E D_{\hat{t}}\right)$, therefore, can be estimated as the product of $0.492 \pm 0.047$ and 1,915 \pm 174 .

To estimate the number of eggs actually deposited in the streambed, it is necessary to parameterize a density-dependent relationship between $P E D$ and the total number of eggs actually deposited in the gravel ([AED]; McNeil 1964). Estimates of $P E D_{\hat{t}}$ and corresponding values of $A E D_{\hat{t}}$ reported for Sashin Creek pink salmon between 1966 and 1976 plus values reported in 1981 were fit to a 3-parameter lognormal curve (Bradford 1995) to describe the relationship between the $\log$ (base 10) of $A E D_{t}$ and $P E D_{t} / 10^{6}$. The curve was fit by least-squares nonlinear regression using the MarquardtLevenberg algorithm as implemented in SigmaPlot ${ }^{\circledR}$ version 8.0. The resulting relationship based on 12 observations predicts the number of eggs deposited in the gravel in year $t$ $\left(A E D_{\hat{t}}\right)$ from the following:

$$
\log \left(A E D_{\hat{t}}\right)=7.531 \exp \left\{-0.5\left[\frac{\ln \left(\frac{P E D_{\hat{t}}}{308}\right)}{6.77}\right]\right\}+\varepsilon_{t 1}
$$

where $\varepsilon_{t 1}$ is drawn randomly from the distribution of residuals, $\sim \mathrm{N}(0.0,0.186)$.

Values of $A E D_{\hat{t}}$ from Equation 4 were combined with the number of fry observed for the 1953 to 1979 brood years to derive a model from which $s f w_{\hat{t}}$ could be estimated. Estimates of $P E D_{\hat{t}}$ for these years were derived from the product of the reported number of returning females and their average fecundity. The resulting estimates of $A E D_{\hat{t}}$ were combined with the log of the reported number of emigrating fry $\left(F R Y_{t}\right)$ and fit to a 3-parameter lognormal curve (Bradford 1995) of the same form as Equation 4 using the previously described algorithm. The resulting model estimates of the number of migrating fry $\left(F R Y_{\hat{t}}\right)$ based on $A E D_{\hat{t}}$

$$
\log \left(F R Y_{\hat{t}}\right)=6.632 \exp \left\{-0.5\left[\frac{\ln \left(\frac{A E D_{\hat{t}}}{3.12 e^{8}}\right)}{9.31}\right]\right\}+\varepsilon_{t 2}
$$

where $\varepsilon_{t 2}$ is a residual randomly selected from the distribution of residuals, $\sim \mathrm{N}(0.01,0.56)$, resulting from the model fitting. Estimates of $s f w_{\hat{t}}$ subsequently are calculated from

$$
s f w_{\hat{t}}=\frac{F R Y_{\hat{t}}}{A E D_{\hat{t}}}
$$

A similar approach was employed to derive estimates of $s s w_{\hat{t}}$. The reported numbers of fry and the log of adult returns were fit to a 3-parameter lognormal curve (Bradford 1995) to generate a model that could predict adult returns in a given year $\left(A D U L T_{\hat{t}}\right)$ from $F R Y_{\hat{t}}$ Model fitting for the densitydependent relationship between the counts of fry $\left(F R Y_{t}\right)$ and adults reported for Sashin Creek between 1940 and 1980 used the previously described algorithm. The resulting model provided the following estimate for $A D U L T_{\hat{t}}$ :

$$
\log \left(A D U L T_{\hat{t}}\right)=4.58 \exp \left\{-0.5\left[\frac{\ln \left(\frac{F R Y_{\hat{t}}}{6.22 e^{7}}\right)}{9.56}\right]\right\}+\varepsilon_{t 3}
$$

where $\varepsilon_{t 3}$ was drawn randomly from the distribution of residuals, $\sim \mathrm{N}(0.0,0.386)$. Consequently, $s s w_{\hat{t}}$ is found from

$$
s f w_{\hat{t}}=\frac{A D U L T_{\hat{t}}}{F R Y_{\hat{t}}}
$$

Note that

$$
A D U L T_{\hat{t}}=y_{t+2}
$$

The lognormal model used here was chosen over those typically used (Quinn and Deriso 1999) because there were no a prioi expectations for the shape of the relations other than that they should be density dependent (Bradford 1995). In addition, the chosen model ultimately produced lower prediction errors than typically used models, such as using the mean or Beverton-Holt, Ricker, or Schnute-Deriso functions. In all cases, curve fitting conformed to the requirements of normality and homoscedasticity. Predictors in the models always described more than $50 \%$ of the error in response variables $(p<0.001)$ and adjusted correlations were $>0.8$ for the first 2 models and $>0.5$ for the model relating fry emigration and adult returns.

Nomenclature-A nomenclature is required to simplify description of the procedures employed in both model validation and analysis of exposure effects. Seeding the model with a value of $y_{t}$ and working through the functions to estimate $y_{t+2}$ is called a calculation. Iterating the calculation recursively 35 times is called a simulation, because it simulates the Sashin Creek pink salmon population over 35 generations. The resulting median number of returns represents the equilibrium population size predicted by the simulation. Likewise, the median number of returns per spawner is the population growth rate predicted by the simulation. This estimate of population growth rate is equivalent to the Malthusian estimator described by Quinn and Deriso (1999). A set of $n$ simulations is referred to as an experiment because it provides a distribution of $n$ equilibrium size and growth rate predictions. The medians of these 2 distributions are used as the estimated equilibrium population size and intrinsic growth rate under the conditions specified for the experiment. Extinction events occur when the number of returning males or females falls below 1 in a given simulation. The extinction rate is the proportion of simulations with extinctions events occurring in an experiment. Parameters derived from experiments in which none of the population suffers $\mathrm{PAH}$ effects described an unexposed population.

Evaluation of the basic model's reliability-The model's reliability was verified by examining how well it predicted the returns to Sashin Creek in a given year through a series of 4 tests. First, for each of the years that were not used to parameterize the model (i.e., brood year $<1953$ and brood year $>1979$ ), the observed number spawners were used to initiate the model and calculate a predicted number of returns. The calculation was repeated 1,000 times to produce a distribution of predicted returns. The location of the observed number of returns within the distribution of predictions was inspected graphically. Second, a runs test of the differences between the observed number of returns and median of the predictions was used to determine if the number of spawners biased predicted values. The 3rd test determined if the model predictions were better than simply using the observed median to predict returns. The difference between the median of the predictions and observed returns were squared for each year and then summed across years. This summed error was compared to the sum of the squared differences between the observed returns and their overall 


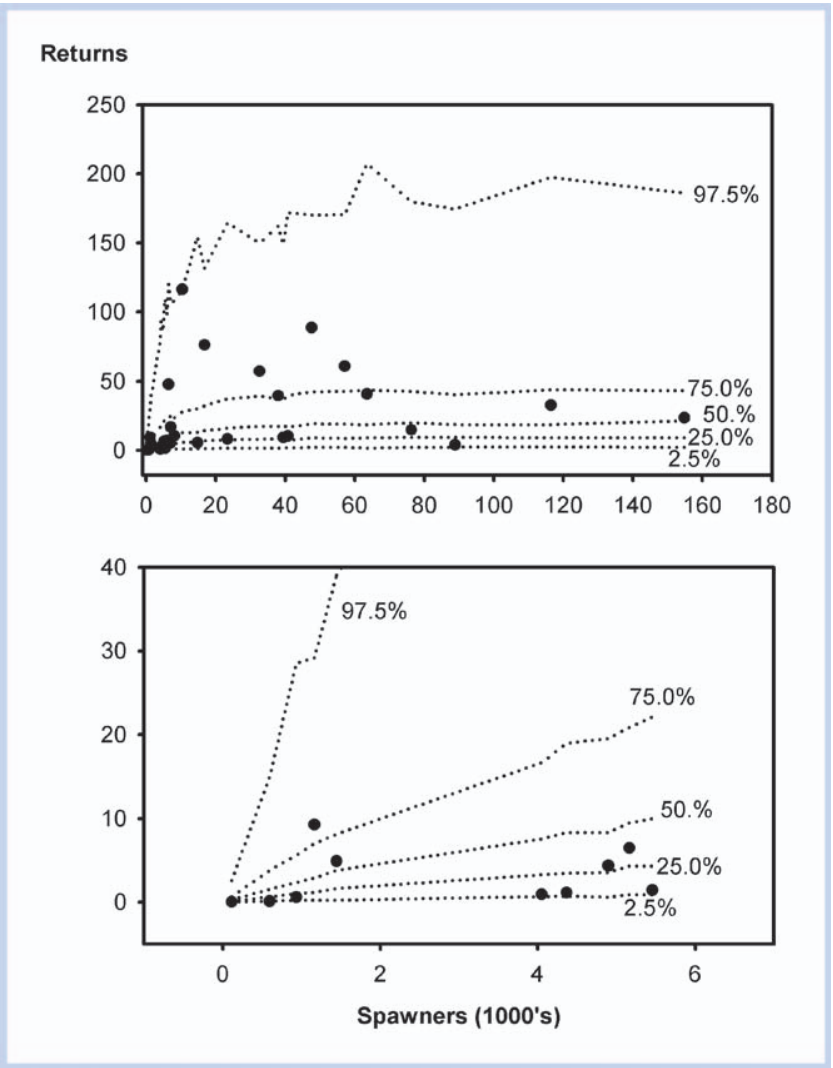

Figure 1. Observed and predicted returns and spawners for Sashin Creek pink salmon. Points depict observed data not used to parameterize the model and lines depict the values associated with various percentiles of the predictions made by the model. Bottom panel expands lower left of upper panel.

median. Finally, a Kruskal-Wallis test was used to compare the median of the observed returns with the median of the predicted medians.

To examine the reliability of a simulation, the equilibrium population size from an experiment was compared to the median of the observed returns using the Kruskal-Wallis test. In this experiment, $y_{t}$ was set equal to 6,100 , the number of salmon that returned in 1935, and then the simulation was repeated 500 times. Each of these procedures also was applied to estimates of growth rate. The even-year run was not tested because it was purposely extirpated in the mid-1950s and restored by transplanting fish from another stream in 1964.

Comparison of basic model predictions with observed data-In general, the simulated population produced estimated returns that were within the range described by the observed numbers of returns. Two of the 30 unused observations have returns that fall outside of the central $95 \%$ of their associated predictions and all of the observed data fall within the central $96 \%$ of the predictions (Figure 1). Eighteen (60\%) of the 30 unused observations are less than their associated median predictions and $40 \%$ are greater. Half of the unused observations fall within the central $50 \%$ of their associated predictions (Figure 1). Thus, the predicted medians are reasonable approximations of the unknown medians. A runs test $(p=0.920)$ indicated an absence of bias in the residuals with observed values randomly falling above or below the median, regardless of the number of spawners. No statistical difference was found between the observed returns and their predicted medians $(p=0.747)$. The overall median return reported for the unused observations is 8,715 compared with

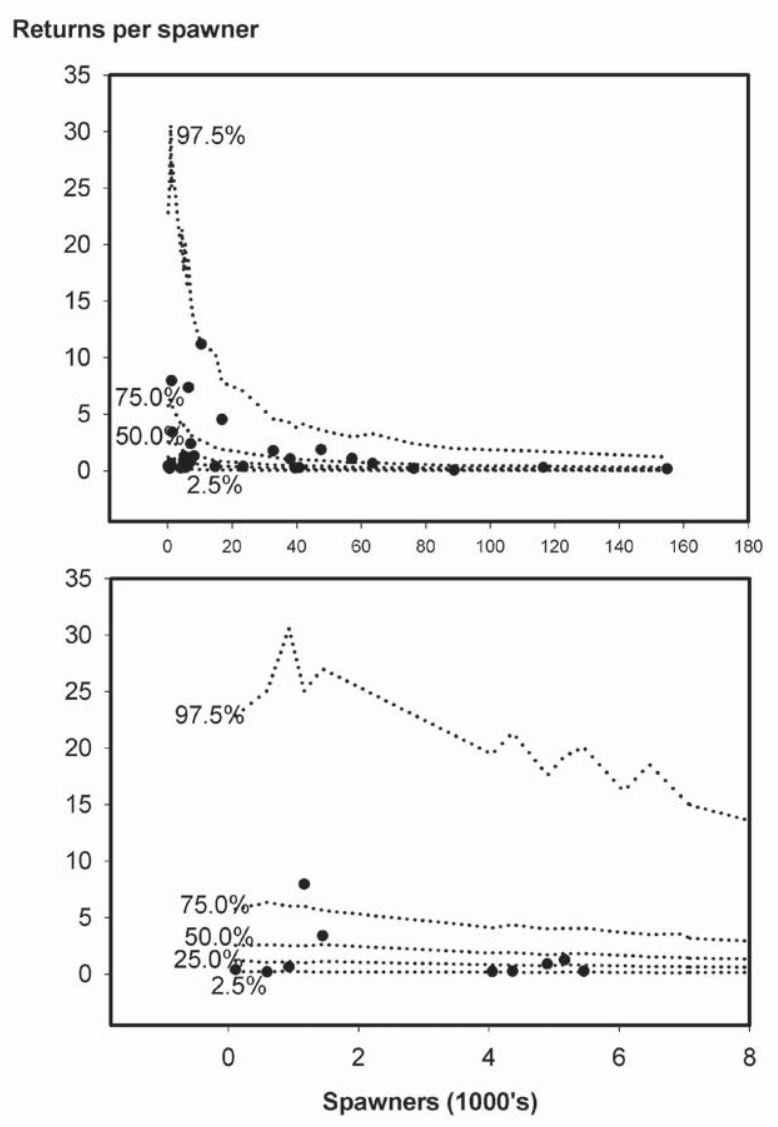

Figure 2. Observed and predicted returns per spawner and spawners for Sashin Creek pink salmon. Conventions as in Figure 1.

10,586, the median of predicted medians. The sum of squared differences between the observed returns and their predicted medians was $19 \%$ lower than the sum of squared differences between the observed returns and their overall median.

Similar responses were observed in the estimated growth rate as measured by the numbers of returns per-spawner (Figure 2). As with the predicted returns, all of the observed returns per spawner fell within the central $96 \%$ of the predicted values. A runs test indicated that there was no bias in the prediction values relative to spawner numbers $(p=$ 0.582), and the observed median number of returns per spawner (0.64) did not differ from the median of the predicted medians ( $1.34[p=0.218])$. In addition, the sum of squared differences between the observed returns per spawner and their predicted medians was $15 \%$ lower than the sum of squared differences between the observed values and their overall median.

Comparison of predicted population parameters with observed parameters-Comparison of the median number of returns and returns per spawner between the odd-year line and the model predictions indicated that the model accurately described both the size and growth of the Sashin Creek pink salmon population. The median number of returns represents the equilibrium population size. Between 1933 and 1999, the median number of returns in the odd-year line was 16,380 pink salmon. The model accurately predicted this size with a median of 12,351 ( $p=0.127)$. Similarly, measures of the median number of returns per spawner provided an accurate 


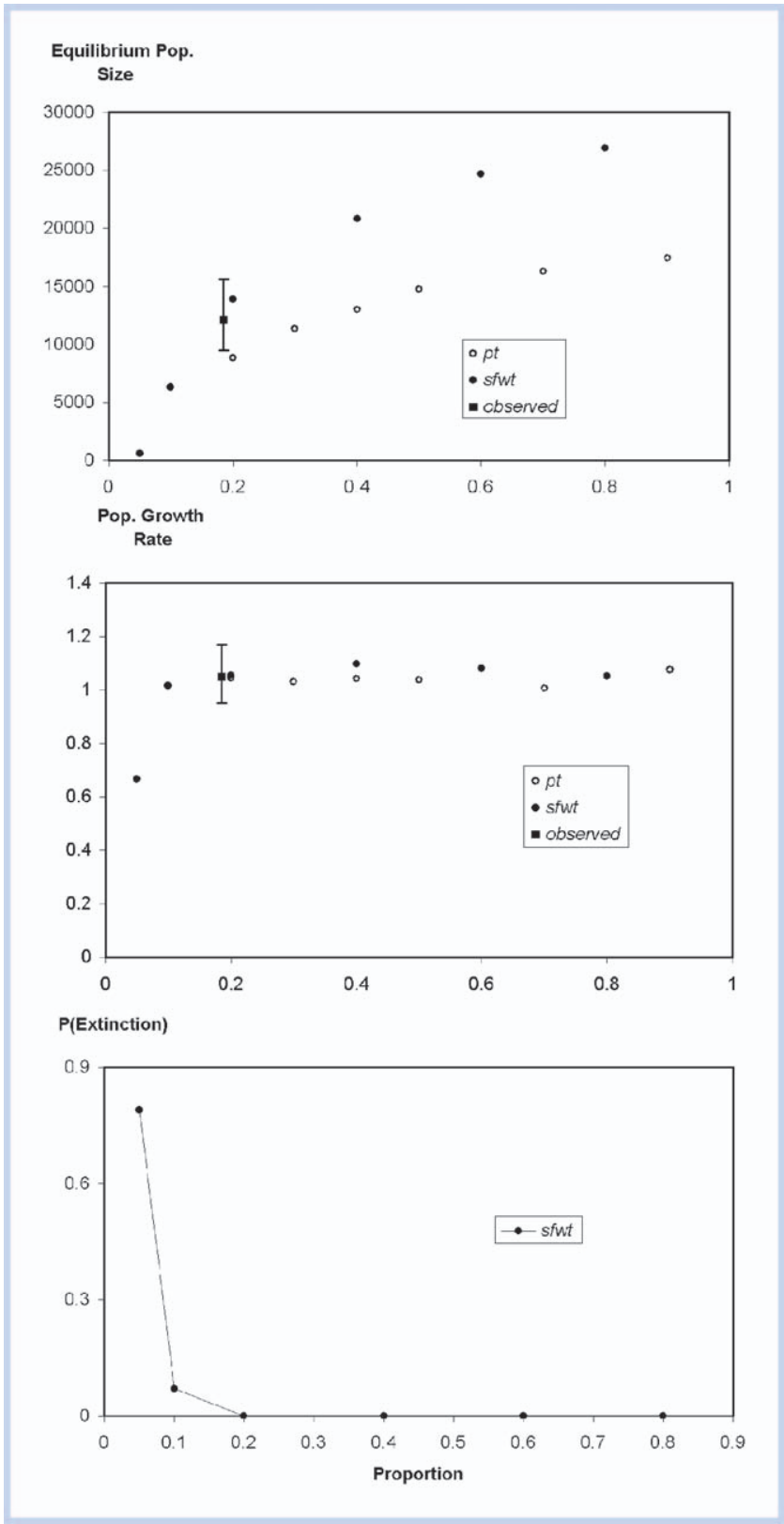

Figure 3. Population parameters resulting from fixing either the proportion of eggs delivered to the stream that are deposited in the gravel $\left(p_{t}\right.$, Eqn. 3$)$ or survival during incubation (sf $w_{t}$, Eqn. 3) and allowing remaining functions to vary. Symbol with error bars depicts the interquartile range of values obtained when all functions are allowed to vary (i.e., observed response). The probability of extinction (P[extinction]) was constant and equal to zero for the population in which all functions vary and for all values of $p_{t}$.

estimate of the intrinsic growth rate of the population. The odd-year line of pink salmon in Sashin Creek has a median growth rate of 1.04 , which is equivalent $(p=0.564)$ to 1.06 , the value predicted by the model. It should be noted that half of the values used for these comparisons were included among those used to parameterize the model.

Sensitivity of the basic model to variation in survival functions - The basic model was manipulated to examine the sensitivity of an unexposed population to variation in each of the three density-dependent functions. In the initial experiment, the value of $p_{t}$ was fixed at a constant value and the remaining functions were allowed to vary in each of 200 simulations. Six total experiments were run in this manner,

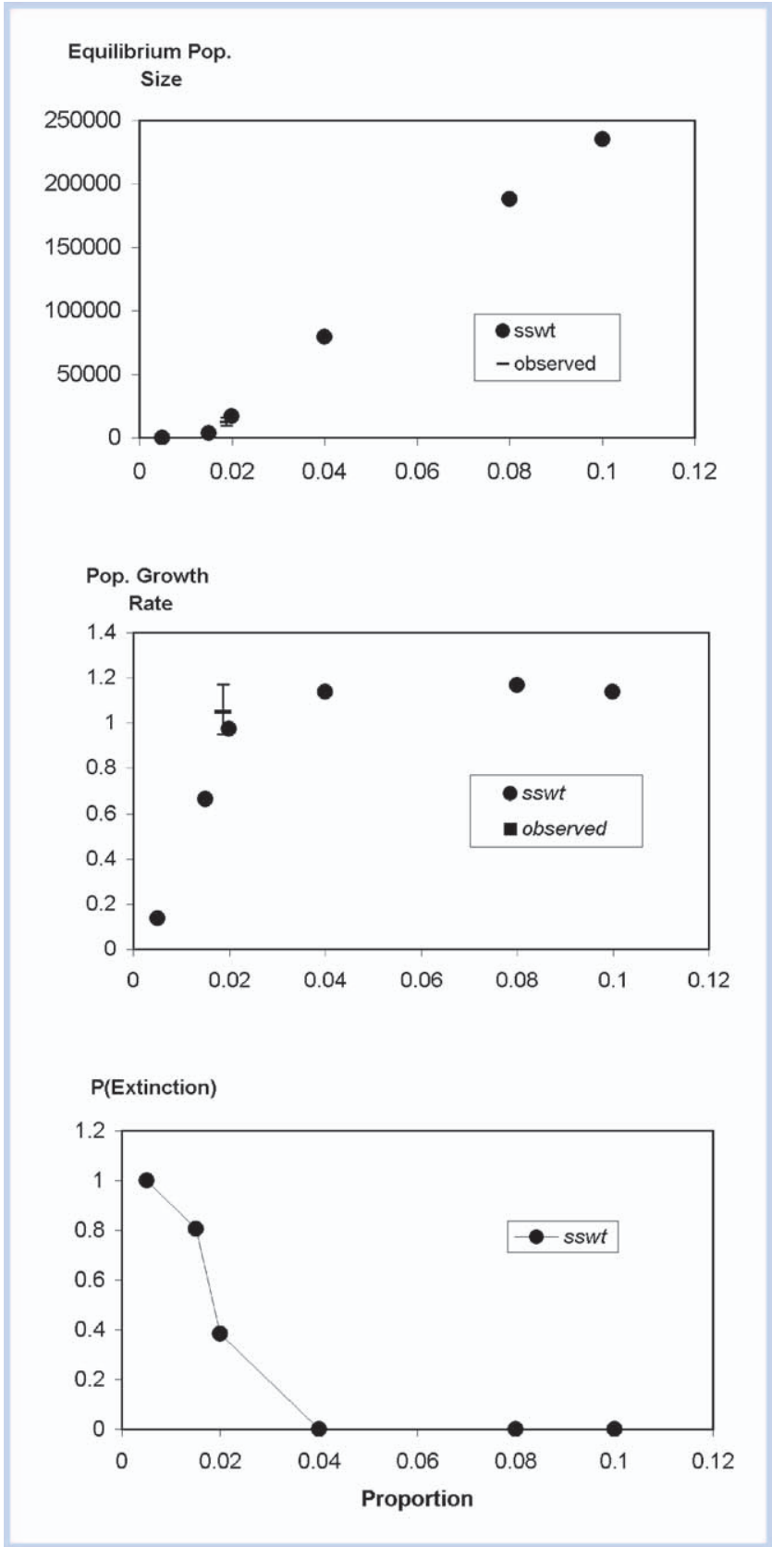

Figure 4. Population parameters resulting from fixing marine survival (sswt, Eqn. 3) at different values. Symbol with error bars is as in Figure 3. Note differences in scale between these panels and Figure 3.

each with $p_{t}$ fixed at a value ranging between 0.2 and 0.9 . At the end of each experiment, the equilibrium population size, population growth rate, and probability of extinction were recorded. A similar procedure was performed by fixing $s f w_{t}$ and $s s w_{t}$. Fixed values for $s f w_{t}$ and $s s w_{t}$ were chosen so that the observed value for an unexposed population fell in the middle of the range.

Unexposed populations were relatively insensitive to changes in $p_{t}$ and $s f w_{t}$. Varying $p_{t}$ had relatively little effect on estimates of equilibrium population size, growth or probability, or extinction (Figure 3). As $p_{t}$ increased from 0.2 to 0.9 , the equilibrium population size increased from 8,824 to 21,567 and the size of an unexposed population that was allowed to vary over all functions was 12,351. Growth varied from 1.04 to 1.08 over the same range of values, 
Table 1. Reported effects on survival for pink salmon during incubation (efw) and marine migration (esw) following embryonic exposure to oil dissolved in water (See Eqn. 10). TPAH = total polynuclear aromatic hydrocarbons; SD = standard deviation

\begin{tabular}{|l|c|c|c|}
\hline & \multicolumn{2}{|c|}{$\%$ Reduction from control } \\
\hline $\begin{array}{l}\text { TPAH concentration } \\
(\mathrm{nL} / \mathrm{L})\end{array}$ & $\begin{array}{c}\text { Fertilization to emergence } \\
(\mathrm{efw})\end{array}$ & $\begin{array}{c}\text { Emergence to maturity } \\
(\text { esw) }\end{array}$ & Heport \\
\hline 18.0 & 23.1 & 15.46 & Heintz et al. 1999 et al. 2000 \\
\hline 19.4 & Not reported & 38.46 & Heintz 2001 \\
\hline 13.3 & 15.23 & 53.85 & Carls et al. 2005 \\
\hline 16.5 & 7.51 & Not reported & - \\
\hline Average & 15.3 & 36.3 & - \\
\hline SD & 7.8 & 19.3 & - \\
\hline
\end{tabular}

compared with 1.06 in the population in which all functions varied. Probability of extinction remained at 0 over all values of $p_{t}$, indicating no effect. The basic model was slightly more sensitive to changes in $s f w_{t}$. The median $s f w_{t}$ in an unexposed population was $18.5 \%$. Fixing $s f w_{t}$ at $40 \%$ increased equilibrium population size to 20,858. Reducing $s f w_{t}$ to $10 \%$ reduced the population size to 6,287 . Population growth only fell below 1.0 when $s f w_{t}$ was reduced to $5 \%$, where extinction probability reached a maximum value of $75 \%$.

In contrast, parameters for unexposed populations were very sensitive to variation in $s s w_{t}$. The median $s s w_{t}$ in the population in which all functions were allowed to vary was $1.87 \%$. Fixing $s s w_{t}$ at $4 \%$ increased the equilibrium population size more than sixfold to nearly 80,000 (Figure 4), though reducing $s s w_{t}$ to $1 \%$ decreased the population size to about 2,000. Growth rate was less sensitive, maintaining relatively constant levels until $s s w_{t}$ fell below $2 \%$. At that point, growth became highly sensitive to further reductions in survival. This translated to an increase in the probability of extinction when marine survival decreased to less than $2.0 \%$.

\section{Incorporation of PAH effects into basic model}

Incubation in PAH-contaminated habitats results in a variety of effects, which ultimately prove lethal to pink salmon. Exposure incurred during incubation can manifest decreased survival during incubation (Marty et al. 1997, Heintz et al. 1999, Carls et al. 2005) and sublethal effects that lead to decreased survival in the marine phase (Heintz et al. 2000). In each of the studies referenced above, exposure took place during incubation in gravel contaminated with Alaska North Slope crude oil. This design simulated the experience of pink salmon eggs developing in streambeds contaminated with oil following the Exxon Valdez oil spill. Examination of the hydrocarbons on the gravel, in water and tissue, by gas chromatography/mass spectrometry revealed that injuries resulted from exposure to 3- and 4-ring PAHs dissolved in water (Heintz et al. 1999). The absence of phytane in tissue or water samples verified that exposures resulted from dissolved species rather than particulates (Marty et al. 1997).

In an exposed population, the number of returns will be

$$
y_{t+2}^{\prime}=y_{t}\left(p_{t} \times f_{t} \times e f w_{t} \times s f w_{t} \times e s w_{t} \times s s w_{t}\right)
$$

In this expression, $p_{t}, f_{t}, s f w_{t}$, and $s s w_{t}$ represent the same processes experienced by individuals in the population if they were not exposed to PAHs. The terms $e f w_{t}$ and $e s w_{t}$ represent the PAH-related decreases in survival in the freshwater and saltwater phases, respectively. Pink salmon returning to a given stream can include a mix of intertidal and freshwater spawners. If some of the returning adults select spawning locations upstream from the PAH source, then exposure will be limited to a portion of the population $\left(o_{t}\right)$. The total number of returns $\left(Y_{t+2}\right)$ will be equal to the sum of the returns from the unexposed and exposed portions of the population

$$
Y_{t+2}=y_{t+2}+y_{t+2}^{\prime}
$$

Combining Equations 3, 10, and 11 yields

$$
Y_{t+2}=s_{t}\left(\left[y_{t}-o_{t} \times y_{t}\right]+\left[o_{t} \times y_{t} \times e f w \times e s w\right]\right)
$$

Recall from Equation 2 that $s_{t}$ is a product of fecundity, the proportion deposited in the gravel, survival during incubation and marine migration.

Parameterization of $P A H$ effects-Oil effects on survival during incubation and marine migration are expressed as proportional decreases to the predicted survival in the absence of exposure. Expression of effects relative to controls removes the assumption that oil effects are independent of other effects on survival because it reflects additional mortality imposed by oil. Thus, for $o_{t} y_{t}$, the number of eggs exposed to PAHs, $s f w_{t}$, and $s s w_{t}$ are decremented by the random variables efw and $e s w$, respectively (Eqn. 10). The decrement due to $e f w$ is $0.847 \pm 0.078$, which is the average ( \pm 1 standard deviation) reduction in survival relative to controls in the reports described in Table 1. Similarly, the decrement due to esw is $0.637 \pm 0.191$ (Table 1). The reported studies were carried out in a hatchery located in the Sashin Creek drainage using pink salmon broodstock collected from a nearby stream. During those studies, salmon were incubated in oil-contaminated gravel. Freshwater survival was estimated as the number of eggs surviving incubation. Survivors were marked and released using standard hatchery methods. Saltwater survival was estimated as the proportion of marked fish that were captured when adults returned to spawn. See references in Table 1 for details.

Experiments to examine effects of $\mathrm{PAH}$ exposure rateExperiments designed to examine the effects of increasing the proportion of the population exposed to the PAH effect followed the same basic procedure as that of the unexposed population. Initially, $y_{t}$ was set to $6,100, o_{t}$ was set to $10 \%$, 500 simulations were generated, and the results tabulated. Thus, the lst experiment examined the effects of chronically 


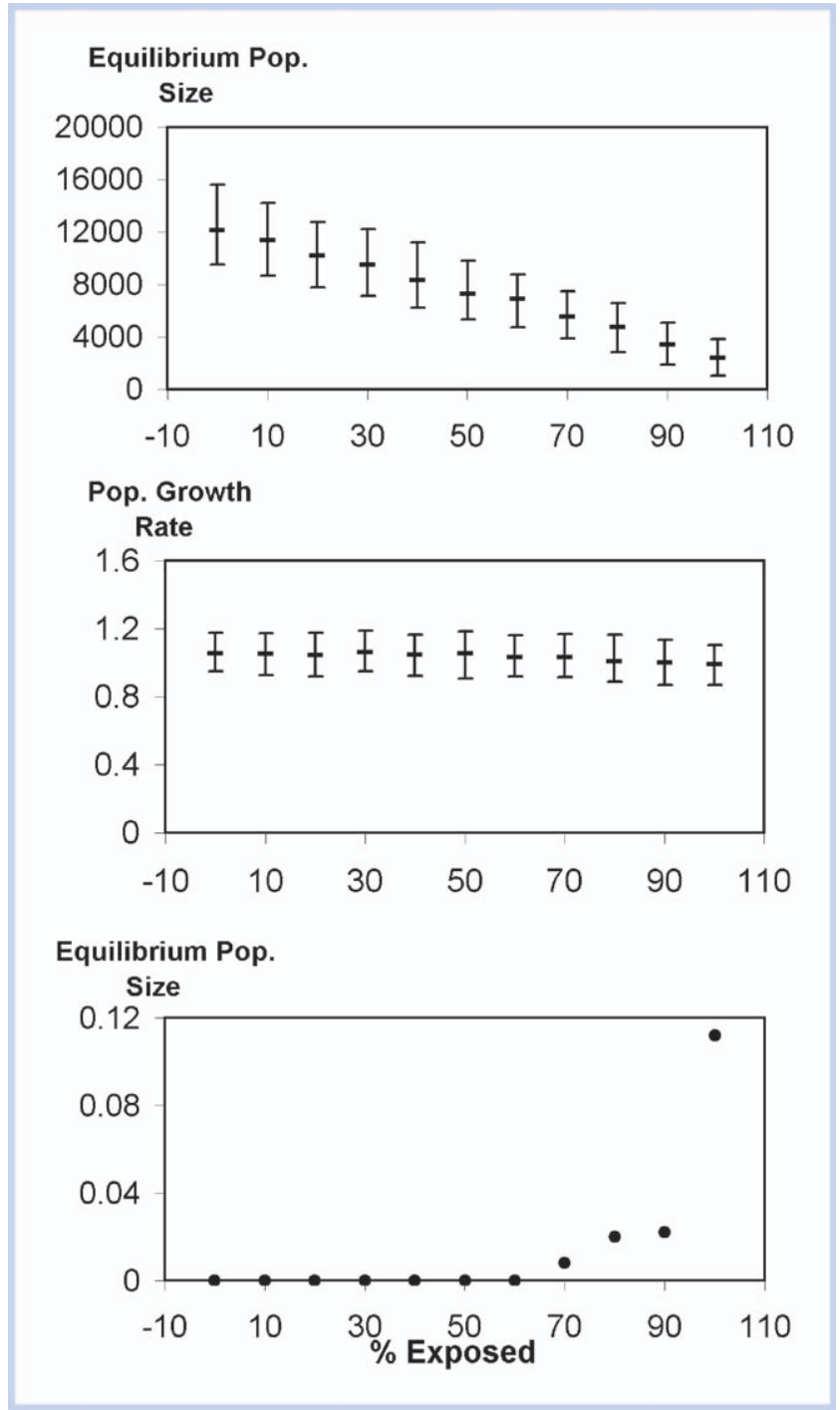

Figure 5. Effect on equilibrium population size, population growth, and probability of extinction (P[extinction]) from an increasing proportion of the population exposed as embryos to PAHs. Symbols depict median and interquartile range of responses. No error estimates are available for $\mathrm{P}$ (extinction).

exposing $10 \%$ of the population to an $18-\mathrm{nL} / \mathrm{L}$ exposure. Experiments were repeated 9 more times, incrementing $o_{t}$ by $10 \%$ each time. The equilibrium population size and growth rates resulting from each experiment were compared with the medians obtained for the unexposed experiment by the Kruskal-Wallis test of medians. The lst comparison involved the experiment in which $o_{t}$ was set to $10 \%$, comparisons continued until a significant difference in medians was detected. The effect of increasing the proportion of the population exposed to the PAH effect on the probability of extinction was examined graphically.

Increasing the percentage of the population exposed to the $\mathrm{PAH}$ effect resulted in a linear decline in equilibrium population size (Figure 5A). The unexposed model populations produced a median size of 12,136 pink salmon. The equilibrium size decreased by $5 \%$ when $10 \%$ of the population was exposed $(p=0.003)$ and reached a $50 \%$ reduction when $70 \%$ of the population was exposed. When $100 \%$ of the population was exposed to the PAH effect, the size dropped by $80 \%$ to 2,373 .
Table 2. Values used in experiments designed to examine effect of decreased relative survival on population parameters. Values of efw and esw are presented as percentages ( \pm 1 standard deviation) of the unexposed survival rates; see Equation 10

\begin{tabular}{|l|c|c|}
\hline Relative survival & efw & esw \\
\hline 1.0 & $84.7 \pm 7.8$ & $63.7 \pm 19.3$ \\
\hline .95 & $80.7 \pm 7.4$ & $60.7 \pm 18.4$ \\
\hline .90 & $77.0 \pm 7.1$ & $57.9 \pm 17.5$ \\
\hline .87 & $73.7 \pm 6.8$ & $55.4 \pm 16.8$ \\
\hline .83 & $70.6 \pm 6.5$ & $53.1 \pm 16.1$ \\
\hline .80 & $67.8 \pm 6.2$ & $51.0 \pm 15.4$ \\
\hline .77 & $65.2 \pm 6.0$ & $49.0 \pm 14.8$ \\
\hline .62 & $52.9 \pm 4.9$ & $39.8 \pm 12.1$ \\
\hline .31 & $26.5 \pm 2.4$ & $19.9 \pm 6.0$ \\
\hline
\end{tabular}

Initially, median growth rate was unaffected by exposure, but once the exposure rate exceeded $60 \%$, it began declining (Figure 5B). The unexposed population had a median growth equal to 1.06 returns per spawner. As noted above, this value is consistent with the observed value for the Sashin Creek odd-year run. In addition, it is within the range of estimates presented by Halupka et al. (1995) when pink salmon populations have been monitored for at least 5 y. Growth remained at this level until $60 \%$ of the population was exposed $(p=0.036)$. After this point, growth began decreasing linearly with increasing exposure. When $100 \%$ of the population was exposed, growth declined to 0.99, indicating the population was no longer self-sustaining.

Similar to growth, the probability of extinction demonstrated a nonlinear relationship with increasing exposure. The population was stable, with extinction probabilities equal to 0.0 until $70 \%$ of the population was exposed. At this point the probability of extinction over the course of 35 generations was $1 \%$ (Figure 5C). Extinction probability then began doubling with each $10 \%$ increase in exposure, resulting in an $11 \%$ probability of extinction within 35 generations when $100 \%$ of the incubating population was exposed.

Experiments to examine the effects of relative survival-The effect of decreased survival due to PAH effects was examined through a series of experiments similar to those described previously. The studies described in Table 1 were designed to determine the lowest effective levels of PAH exposure on pink salmon embryos. However, it is feasible that exposure to other chemicals, higher levels of PAHs, or synergisms could exacerbate the observed effects and further decrease survival beyond the levels identified in the toxicity studies. A set of 11 experiments, each incorporating different values for $o_{t}$, was repeated for 8 levels of survival relative to those described in Table 1. The mean and standard deviations of efw and esw were decreased in units of approximately 5\% for each experiment until efw and esw were reduced by approximately $25 \%$ (Table 2). The next 2 experiments involved reductions of approximately $60 \%$ and $70 \%$ (Table 2). For the purposes of discussion, relative survival for a given experiment is the proportional reduction in $e f w$ and $s f w$ relative to the average values observed when eggs were exposed to aqueous total 


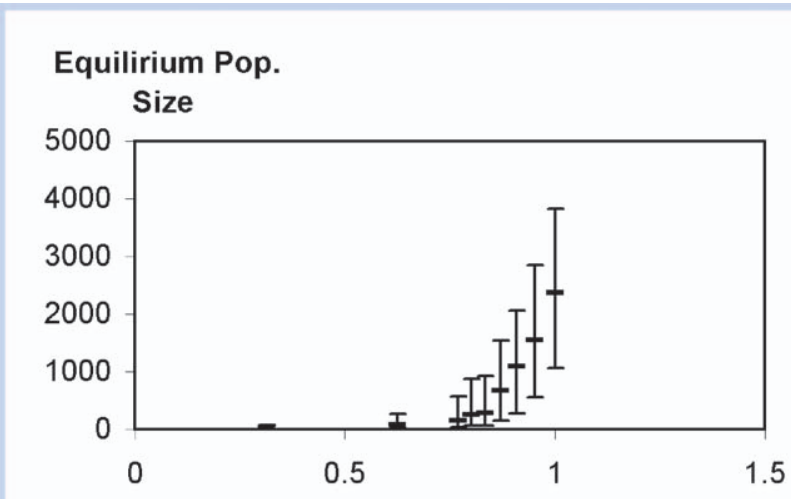

Pop. Growth Rate

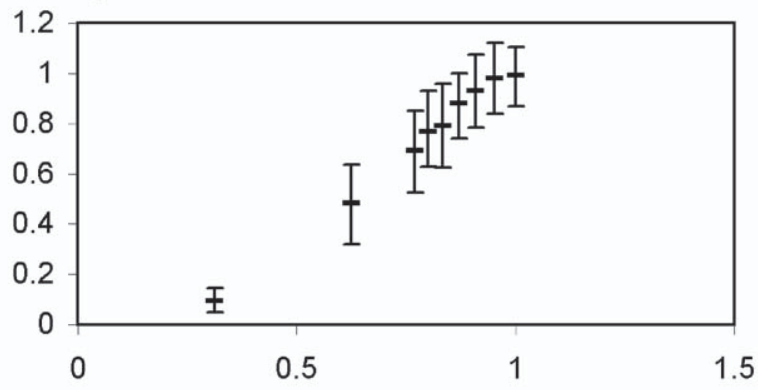

$\mathrm{P}$ (Extinction)

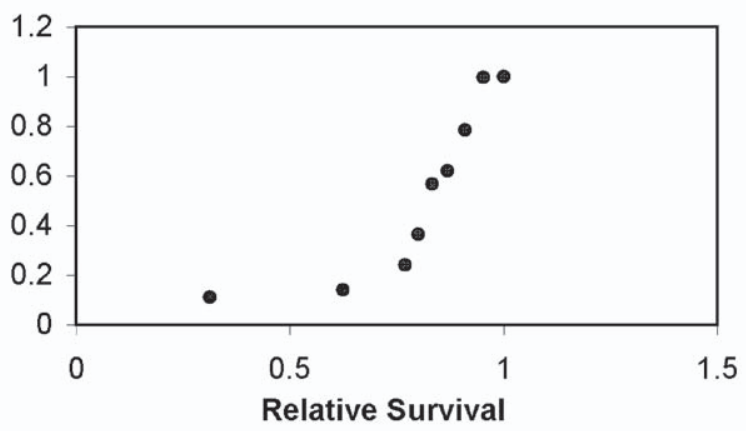

Figure 6. Equilibrium population size, population growth, and probability of extinction when $100 \%$ of the pink salmon embryos are exposed to the decreasing survival relative to the observed PAH effect. A relative survival of 0.90 indicates efw and sfw were set to $90 \%$ of the value observed in toxicity assays where pink salmon eggs were exposed to an aqueous PAH concentration near $18 \mathrm{~nL} / \mathrm{L}$. Symbols as in Figure 5 .

PAH concentrations near $18 \mathrm{~nL} / \mathrm{L}$ (Table 1). Thus, a relative survival of $30 \%$ indicates that the values of efw and $s f w$ were set to $30 \%$ of the values observed in toxicity assays (Table 2). The equilibrium population size for each combination of $o_{t}$ and relative survival was plotted to examine the interaction between relative survival and exposure rate. Similar plots were developed for the population growth rate and probability of extinction.

Small decreases in relative survival placed severe limits on the buffering capacity offered by density dependence when $100 \%$ of the population is exposed. Decreasing survival due to exposure produced a nonlinear decrease in equilibrium population size (Figure 6A). Initially, decreased population size was linearly related to relative survival for small increments until efw and esw are each reduced by $16 \%$. At this point the rate at which population size declined was greatly reduced, but the population size was reduced to less than $3 \%$
Growth / Extinction probability

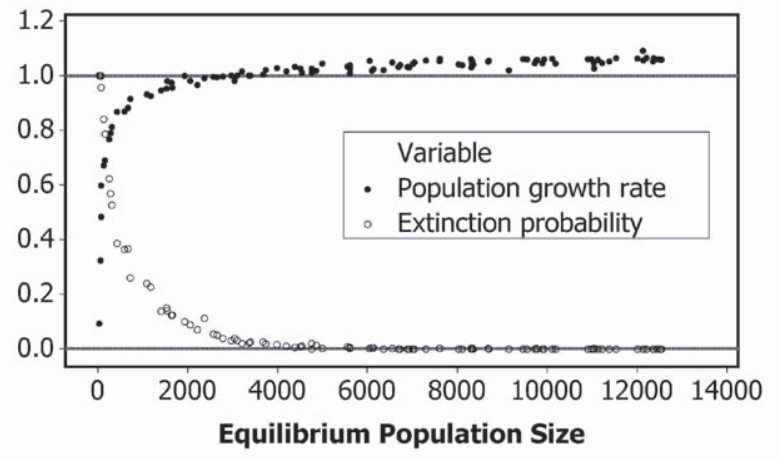

Figure 7. Observed growth and extinction probabilities from all experiments performed on a simulated pink salmon population.

of the unexposed size. A similar pattern was observed in population growth. Initially, there is a rapid linear decline in growth as efw and esw decrease, but once they were reduced by $37 \%$, the rate at which growth rate declined decreased (Figure 6B). However, at this point, the population was no longer stable. Growth was less than one-half, indicating that the population was declining by $50 \%$ with every generation. Consequently, the probability of extinction increased to 99.8\% (Figure 6C).

Trade-offs between growth and extinction probability at different levels of population size demonstrated the buffering capacity offered by density dependence (Figure 7). Extinction probabilities only became detectable when population size fell to about half its initial level. At that point, growth still exceeded one return per spawner. Thus, so long as the population size was more than half the unexposed level, increased mortality resulting from toxic effects was compensated by density-dependent responses, which maintained growth. However, if the population size fell below $50 \%$ of the unexposed level, then stability decreased as was demonstrated by a rapid increase in the probability of extinction. Note that the population experienced a small extinction risk $(<5 \%)$ before growth fell below 1.0. This indicates that, once population size fell to levels near 3,500, chance began to influence population stability. Beyond this point, the toxic effects eroded growth to the degree that the population was not longer self-sustaining and extinction probabilities increased rapidly.

Interaction between exposure rates and relative survivalExamination of the interaction between the PAH effect on survival and exposure rate demonstrated the capacity of the population to accommodate increased exposure and decreased survival (Figure 8). Decreases in survival associated with PAH exposure or increased exposure rate resulted in a linear reduction in population size until the exposure rate exceeded $50 \%$. At this point the population began to fall below $50 \%$ of the initial size and PAH effects began interacting with exposure rate in a more than additive fashion. For example, when $50 \%$ of the population was exposed, population size decreased by about $40 \%$ as relative survival declined from 1.0 to 0.625 . In contrast, the same decrease in relative survival resulted in a $55 \%$ decrease in population size when an additional $10 \%$ of the population was exposed. At the most extreme levels of exposure and 


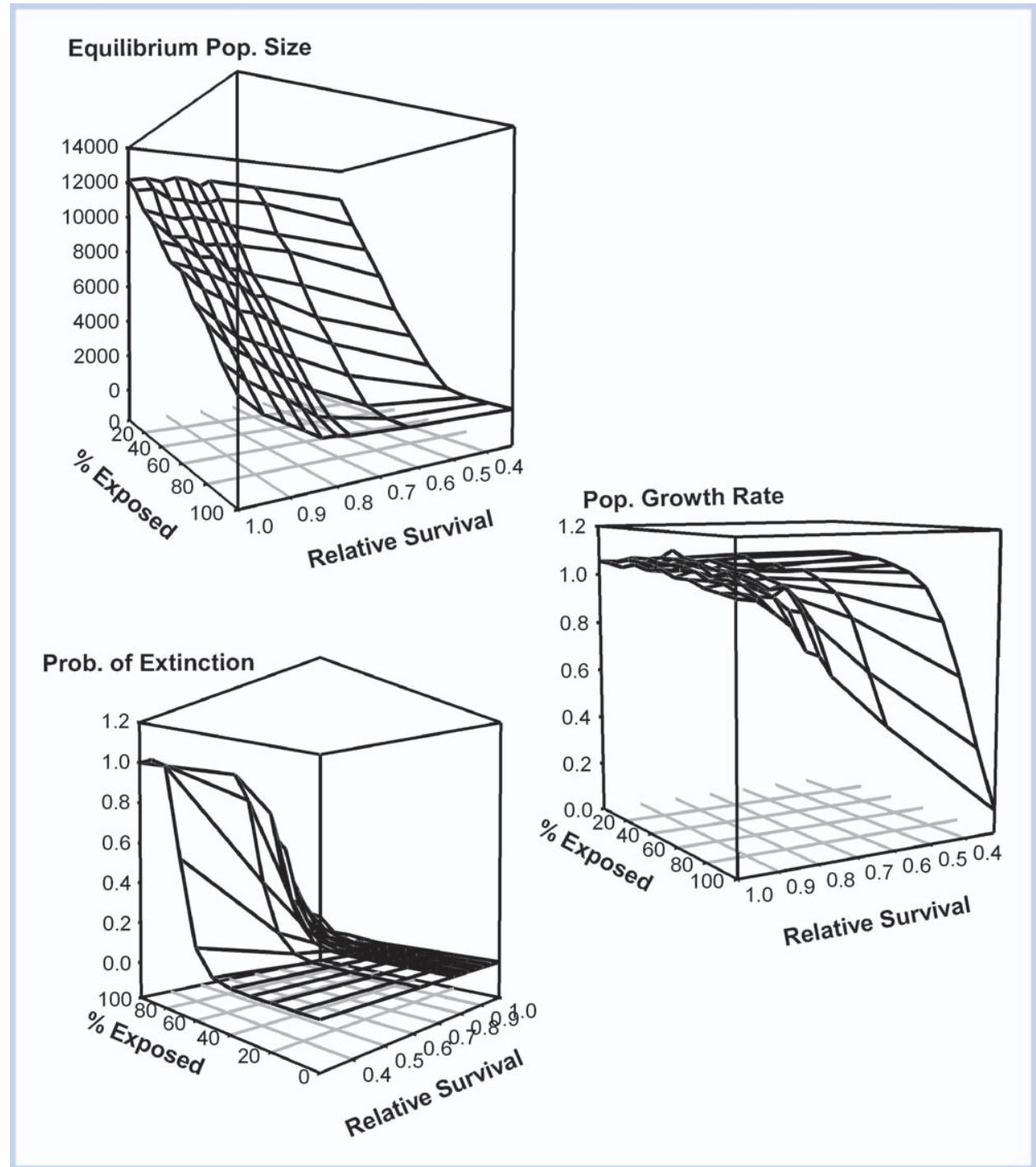

Figure 8. Predicted equilibrium population size, growth rate, and probability of extinction resulting from the interaction between exposure rate and relative survival. Relative survival is expressed as in Figure 6 . Note $x$ - and $y$-axes of the probability of extinction plot are scaled differently from the other 2 plots.

relative survival, this interaction resulted in population sizes that were less than $1.0 \%$ of the unexposed size.

Population growth was more resistant to the interaction between exposure rate and relative survival than population size. Only the greatest degree of contamination or the lowest relative survivals decreased growth to less than 1 return per spawner (Figure 8). When less than $50 \%$ of the population was exposed, growth was maintained at levels greater than 1.0 regardless of the relative survival. However, once exposure rates exceeded $60 \%$, they began interacting with relative survival to drive growth to levels below 1.0 return per spawner at exponential rates.

The persistence of growth rates greater than 1.0 when exposure rates were less than $50 \%$ means that there was little risk of extinction at most levels of relative survival, despite reduced population size (Figure 8). Population stability was maintained when $50 \%$ or fewer of the population was exposed to PAHs and relative survival was $77 \%$. At this point the probability of extinction was still less than $1 \%$, growth was 1.03 , and the equilibrium population size was 5,600 , approximately one-half the unexposed size. Increasing either the extent of exposure at this relative survival, or the relative survival at this extent resulted in increased probabilities of extinction. Thus, this was the last point at which stability of the population reasonably could be guaranteed. The lowest point at which extinction was guaranteed was when exposure rate was $90 \%$ and relative survival was $31 \%$. However, the population had more than a $50 \%$ probability of extinction when exposure rates were $100 \%$ and relative survival was $83 \%$.

Effect of removing efw and esw-Values of efw and esw (Eqn. 10) were manipulated to determine how injury at different life stages affects equilibrium population size, population growth, and stability. The lst experiment involved setting sfw to 0 and examining population parameters after 200 simulations when $100 \%$ of the population was exposed to the PAH effect with a relative survival of 1.0. Experiments were repeated for relative survivals of $0.9,0.87,0.83,0.62$, and 0.31 . A similar set of experiments were conducted in which $e f w$ was set equal to 0 and $s f w$ was allowed to vary. The resulting values of equilibrium population size, growth, and stability were plotted against relative survival.

$\mathrm{PAH}$-induced reductions in marine survival had a greater impact on population parameters than reductions in fresh- 


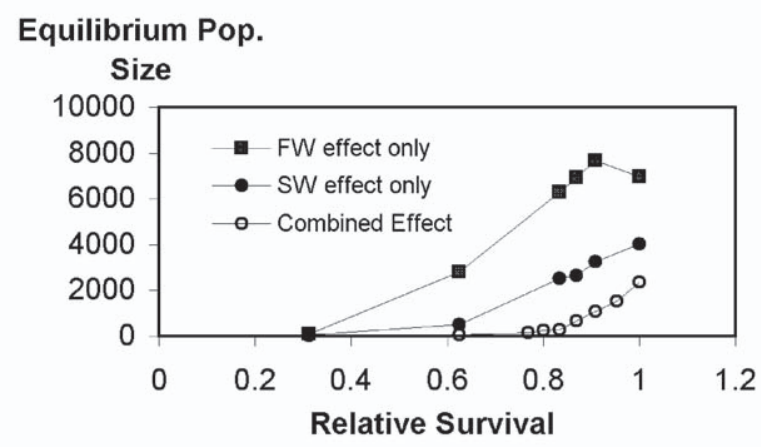

Pop. Growth Rate

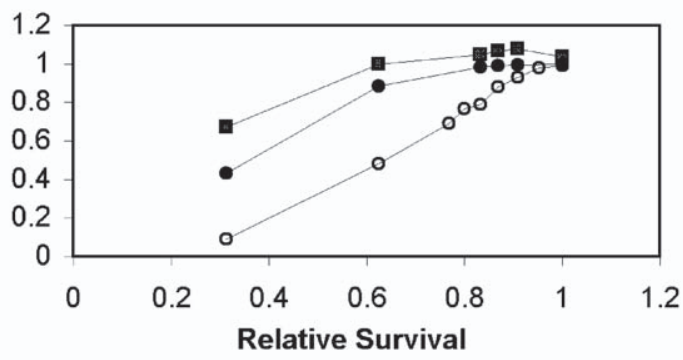

$P($ Extinction)

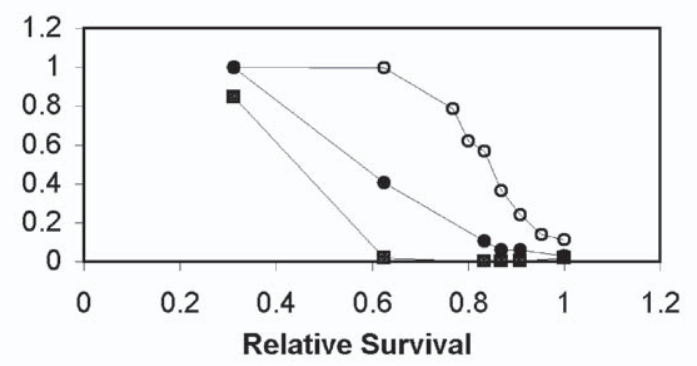

Figure 9. Population parameters at different levels of relative survival after removing PAH effect from freshwater (efw, see Eqn. 10) and marine surviva (esw, see Eqn. 10). All data reflect experiments in which $100 \%$ of the population was exposed to effects; combined effect refers to results when survival is decremented in both the freshwater and marine phase. Lines labeled Marine effect only depict effects when only the marine survival is decremented to reflect PAH exposure. Relative survival is as in Figure 6.

water survival. Recall that exposing $100 \%$ of the population to the PAH effect with a relative survival equal to 1.0 reduced equilibrium population to 2,373 and growth to 0.99 and increased the probability of extinction to $11 \%$ (Figure 5). Removing ssw from the model resulted in reducing equilibrium population size to 6,953 and had no effect on growth or stability (Figure 9). Thus, the only substantial impact on the population resulting from $\mathrm{PAH}$-induced reductions on freshwater survival was reduced population size. In contrast, removal of efw produced population parameters that more closely resembled those of a population experiencing a combination of freshwater and marine impacts. Removal of efw produced an equilibrium population size of 4,033 , a slight reduction of growth, and probability of extinction equal to 3\% (Figure 9).

Examination of population parameter responses in these experiments to decreasing values for relative survival indicated a nonadditive interaction between $e f w$ and $s f w$. Effects in the freshwater phase reduced equilibrium size (Figure 9) relative to an unexposed population, but stability was maintained over a relatively large range of relative survivals. This stability likely resulted from the compensation afforded by density dependence in the marine phase as indicated by the positive relationship between marine and relative survival (data not shown). In contrast, populations that only experience PAH effects in marine phase have reduced stability. For these populations, equilibrium population size is lower, because there is no density compensation to the PAH effect on the number of returning adults. Thus equilibrium population size is lower and stability is decreased, but higher than that of populations experiencing a combination of effects. Apparently, some compensation is afforded by the density dependence of egg deposition rates and freshwater survival, which serves to increase the number of fry entering saltwater in populations that only experience marine effects. This compensation is further reduced in populations that experienced combined effects. Thus, combining efw and $s f w$ further reduces equilibrium population size and amplifies the probability of extinction well above that of the other test populations.

\section{DISCUSSION}

The equilibrium size of the simulated pink salmon population diminished as their natal habitat become increasingly contaminated with PAHs, leading to increased vulnerability to extinction. This process resulted in an $80 \%$ decrease in median return number when $100 \%$ of the population was exposed and a growth rate just below that was required for replacement. Consequently, the primary effect of embryonic exposure to PAHs is to diminish the equilibrium population size. Population growth rate is much less affected, which indicates that, when equilibrium population size is sufficiently large, density dependence compensates for the PAH toxicity. This suggests that exposed populations can recover if the contamination is removed, as was demonstrated by pink salmon populations in Prince William Sound following the Exxon Valdez oil spill (Exxon Valdez Oil Spill Trustee Council 2002). However, these data further demonstrate that, if the equilibrium population is eroded to a small enough number, density-independent processes will overwhelm the compensatory effects afforded by density dependence.

The absence of extinctions in the experiments involving unexposed populations demonstrates the protection offered by density dependence. This protection could be observed up to the point where $50 \%$ of the population was exposed to an environment with a relative survival of 0.77 . Beyond this point, the random errors in the survival functions combine with the small population sizes to produce survivals equal to 0 . Analysis of the relative contributions of different life stages to these results further support the idea that density dependence can compensate for toxic effects in early life stages. Had the toxic effects of embryonic exposure been limited to the freshwater phase, then stability would have been maintained under most conditions. Consequently it is the delayed effects resulting from embryonic PAH exposure that make the effects of PAH so pernicious.

The error terms associated with the density-dependent functions comprise measurement and random errors, the latter resulting from density-independent effects on survival. The contribution of measurement error to this variation is likely quite small. Anadromous pink salmon are relatively 
easy to intercept and count, and strict sampling protocols can be applied to egg deposition surveys. Thus, variation around parameter estimates are likely to be within the same order of magnitude as the point estimates. In contrast, the densityindependent effects are known to be quite large. Observed returns to Sashin Creek vary over 4 orders of magnitude, and this variation is typical of most southeastern Alaska pink salmon populations (Halupka et al. 1995). Despite this wide variability, productivity is maintained in these systems through density-dependent processes (Bradford 1995). However, the data provided in the simulations indicates that, if the equilibrium sizes of these populations were to decline sufficiently, they would be at risk of extinction.

It is important to note that the objective of this effort was to simulate a given pink salmon population while minimizing the residual error. Consequently, the curve fitting procedures used were those found to result in the least amount of residual error, in contrast to more typically used curves. In addition, the error terms incorporating density independence are derived from functions with predictor variables whose errors were unknown, which might have introduced bias (Quinn and Deriso 1999) in the parameter estimates. However, comparison of the resulting error structure with observed data demonstrates that the simulation adequately represents the error structure of the observed data set. For example, the observed coefficient of variation for returns per spawner for the odd-year line is $154 \%$, and the median coefficient of variation from the simulation of the unexposed population is $148 \%$. Combining this simulation with toxicological observations made on fish rearing in the same watershed has provided a powerful model that can be used to examine toxic impacts on a population.

The conclusions drawn here regarding the compensatory effects of density dependence are the same as those reached by others examining toxic effects on exposed populations. The compensatory effects of density dependence in a Chinook salmon population reduced contaminant-related effects on equilibrium population size in a series of life table response experiments (Spromberg and Meador 2005). Linke-Gamenick et al. (1999) described a laboratory study designed to evaluate the relationship between density and flouranthene exposure in capitellid worms. They reported that the negative relationship between population growth rate and PAH concentration could be alleviated by increasing population density. Similarly, Grant (1998) found that density dependence compensated for toxicity in a life table response study involving a copepod exposed to dieldrin. Snell and Serra (2000) developed a density-dependent model for rotifers and found that the population was not sustainable if growth rate was reduced by more than $5 \%$, similar to the $5 \%$ decrease in fitness reported here at the $100 \%$ exposure rate. In both Snell and Serra (2000) and this study extinction risks were between $10 \%$ and $15 \%$. However, extinction risk increased significantly once Snell and Serra (2000) added density-independent effects.

The relative insensitivity of growth rate to the effects tested here suggests growth may be an inappropriate endpoint to toxicity assays designed to examine exposure risk to populations. Linke-Gamenick et al. (1999) note that population growth rate is a convenient endpoint that integrates the potential antagonism between density dependence and toxicity. They argue that it is a valuable metric for understanding how toxicity influences populations, as demonstrated by various workers (e.g., Grant 1998; Spromberg and
Meador 2005). To this end, Nöel et al. (2006) developed an assay method that measures the sensitivity of growth to the interaction between density and toxicity. However, Forbes et al. (2001) note that low-level exposures may not be detectable in populations whose growth rates are depressed by density dependence and hence stability may be underestimated. This point of view is echoed by Grant (1998), who pointed out that growth rate is not a helpful indicator of behavior in populations regulated by density dependence. This was the case described here, once the population growth rates had been reduced by $20 \%$, equilibrium population size already had decreased by $90 \%$, and extinction probability had climbed to more than $30 \%$. Thus other population characteristics likely are more sensitive to contaminant effects. For example, Spromberg and Meador (2005) found Chinook salmon age structure and equilibrium population size to be more sensitive to toxic effects than population growth rate. Similarly, Snell and Serra (2000) found a $20 \%$ reduction in growth rate resulted in a $60 \%$ probability of extinction in rotifers. Therefore, a strict focus on growth rate is likely to underestimate the risks posed by contaminants on wild populations.

The need to incorporate characteristics other than growth in evaluating contaminant risk is problematic. Laboratory studies designed to evaluate population effects often rely on density as an independent variable, and laboratory-based life table response experiments can only project effects in a constant environment (Forbes et al. 2001). For example, Heintz et al. (2000) observed an effect of PAH exposure on individual growth rate in pink salmon, but reduced growth did not affect fecundity among returning adults because slower growing individuals did not return. Under constant conditions in the absence of predators, individual effects on growth likely would have reduced average fecundity in exposed populations. Efforts to combine individual effects with detailed demographic data for wild populations, such as presented by Spromberg and Meador (2005) and Snell and Serra (2000), can provide for more complete assessments. However, obtaining the sort of demographic data required for these analyses is likely to be difficult as is data describing the appropriate individual effects (Schaaf et al. 1993). Moreover, allowances for natural variation will further restrict the number of populations that can be assessed. Consequently, there still needs to be reconciliation between individual and population effects in a way that has meaningful application to wild populations.

The converse issue of detecting effects in existing populations is also problematic. Our ability to detect changes in the equilibrium size and growth rate of wild populations is constrained by the amount of baseline data we have prior to impacts (Pella and Myren 1974). This means that detection of small decreases in equilibrium population size is unlikely in most cases. For example, data presented here indicate that even low levels of contamination can influence equilibrium population size, with no detectable effect on growth. Yet such effects could never be detected because the number of pink salmon returning to Sashin Creek varies over 4 orders of magnitude. This indicates that managers should regard populations known to be in decline with a high degree of concern. Particular concern should be expressed for those populations experiencing contaminant exposure during sensitive life stages or susceptible to a high degree of densityindependent mortality. 
Other reports describing the extreme sensitivity of developing fish eggs to low levels of PAHs indicate these data are applicable to a wide variety of species and life histories. The same effects during incubation reported for pink salmon have been shown to occur at similar exposure levels in larvae of Pacific herring (Clupea pallasi), fathead minnows, rainbow trout (Oncorhynchus mykiss), and mummichogs (Fundulus heteroclitus) (Carls et al. 1999; White et al. 1999; Coulliard 2002; Hawkins et al. 2002; Colavecchia et al. 2004). Insofar as the delayed effects reported for pink salmon represent sequelae resulting from sublethal effects incurred during sensitive developmental stages, it is reasonable to conclude these other species would also experience similar delayed effects. Consequently, the general conclusion that embryonic PAH exposure significantly can reduce the productivity and stability of exposed populations can be extended to these species.

It might be argued that the types of species investigated thus far would be unlikely to experience extinction because they could adapt rapidly to chronic PAH exposure. Adaptation to the effects of chronic pollution has been demonstrated for mummichogs living downstream from the Atlantic Wood creosote plant (Ownby et al. 2002). This plant operated between 1926 and 1990, indicating that 35 generations was sufficient time for mummichogs to increase their resistance to acute doses of PAHs. However, this adaptive tolerance to PAH exposure may result in other costs to fitness (Meyer and Di Giulio 2003). For example, mummichogs living downstream from a kraft pulp mill had altered reproductive behavior relative to those in an uncontaminated estuary (Leblanc et al. 1997). Furthermore, brown bullheads chronically exposed to PAHs in the Black and Schuylkill Rivers (Baumann et al. 1990, Steyermark et al. 1999) demonstrated truncated age distributions relative to those in uncontaminated locations. Consequently, adaptation leading to the ability to withstand acute exposures to a local contaminant does not necessarily guarantee high levels of productivity in the face of chronic exposure.

Furthermore, adaptation would have to respond to other injuries or mechanisms that are not accounted for in this simulation. The studies referenced in Table 1 only account for embryotoxic effects and teratogenic sequelae that manifest reduced marine survival. These studies employed exposures to PAHs that are not associated with narcotic effects (Barron et al. 2004). Nor do the studies referenced in Table 1 include PAH-induced impairments to reproduction, which could contribute significantly to an increase in extinction risk (Levin 1996). Reported impairments to reproduction include reduced offspring survival rates (Bue et al. 1996), impaired reproductive behavior (Le Blanc et al. 1997), and regulation (Van den Heuvel and Ellis 2002). Additional extinction risk may accrue from increased genetic load resulting from increased frequency of mutation (Roy et al. 1999) or genetic damage to reproductive ability (White et al. 1999).

The population effects following embryonic exposure described here derive from environmentally persistent PAHs comprising primarily 2 and 3 rings (Heintz et al. 1999). These same compounds are among those found in urban runoff (Fulton et al. 1993; Zeng and Vista 1997; Menzie et al. 2002). In North America, consumption of oil products accounts directly for the release of 84,400 metric tons of hydrocarbons each year (National Research Council 2002), most of which ends up in estuarine wetlands (Kennish 2002). Leachates from urban areas have been shown to have PAH loads consistent with those found effective in Table 1 (Menzie et al.
2002). Habitat fragmentation has been proposed as an important cause for lost diversity and productivity of aquatic species (Roberts and Hawkins 1999). Apparently, highway runoff can be added to the list of contributing causes to reduced productivity in urbanized estuaries.

Despite evidence of population declines in contaminated aquatic ecosystems, direct linkages between declines and chemical exposure rarely have been identified. The primary reason for this is that the relatively large interannual variation in the recruitment of most aquatic species masks subtle reductions in equilibrium population size. For example, between 1935 and 1979 the Sashin Creek pink salmon run ranged between 600 and 114,400 adults. Thus, it is unlikely that subtle effects on population size would have been detected during that period. Moreover, there are few, if any, comparable data sets. High recruitment variability means that managers typically detect significant declines long after initiation of the trend. By the time these decreases are detected, managers likely are faced with untangling a multiplicity of causative agents. Ascribing declines to contaminants is further impeded because contaminant effects are indirect unless populations face acute exposures. The reductions in marine survival detected for pink salmon exposed during incubation described in Table 1 likely relate to impediments to their individual growth rates (Heintz et al. 2000). Slowgrowing salmon face increased levels of predation and potential starvation in winter (Beamish et al. 2004). Thus, adult salmon that had experienced exposure returned in lower numbers but with the same average size as unexposed adults. Without overt indications of chemical exposure, managers are unlikely to identify contamination as a causative agent to eroding production of urbanized estuaries despite the consequences identified here.

Acknowledgment-This study was completed under funding provided by the Exxon Valdez Oil Spill Trustee Council (restoration study 98476). Thanks are extended to J. Pella, M. Sigler, and T. Quinn for their guidance in model development.

Disclaimer-Use of a commercial product does not represent endorsement by the National Marine Fisheries Service, NOAA. The findings and conclusions in this paper are those of the author and do not necessarily represent the views of the National Marine Fisheries Service.

\section{REFERENCES}

Barron MG, Carls MG, Heintz R, Rice S. 2004. Evaluation of fish early life stage toxicity models of chronic embryonic exposures to complex polycyclic aromatic hydrocarbon mixtures. Toxicol Sci 78:60-67.

Baumann PC, Harshbarger JC, Hartman KJ. 1990. Relationship between liver tumors and age in brown bullhead populations from two Lake Erie tributaries. Sci Total Environ 94:71-87.

Beamish R, Mahnken C, Neville CM. 2004. Evidence that reduced early marine growth is associated with lower marine survival in coho salmon. Trans Am Fish Soc 133:26-33.

Bradford MJ. 1995. Comparative review of Pacific salmon survival rates. Can J Fish Aquat Sci 52:1327-1338.

Bradshaw R, Heintz R. 2005. User manual for the Sashin Creek Weir Database: SCWDATA, 1934-98. Juneau (AK): Auke Bay Laboratory, Alaska Fisheries Science Center, National Marine Fisheries Service, National Oceanic and Atmospheric Association.

Bue BG, Sharr S, Mofitt SD, Craig AK. 1996. Effects of the Exxon Valdez oil spill on pink salmon embryos and pre-emergent fry. Am Fish Soc Symp 18:619627.

Carls MG, Heintz RA, Marty GD, Rice SD. 2005. Cytochrome P4501A induction in oil-exposed pink salmon embryos predicts reduced survival potential. Mar Ecol Prog Ser 301:253-265. 
Carls MG, Rice SD, Hose JE. 1999. Sensitivity of fish embryos to weathered crude oil. Part 1. Low-level exposure during incubation causes malformations, genetic damage, and mortality in larval Pacific herring (Clupea pallasi). Environ Toxicol Chem 18:481-493.

Colavecchia MV, Backus SM, Hodson PV, Parrott J. 2004. Toxicity of retene to early life stages of fathead minnows (Pimephales promelas). Environ Toxicol Chem 23:1709-1718.

Cook PM, Robbins JA, Endicott DD, Lodge KB, Guiney PD, Walker MK, Zabel EW Peterson RE. 2003. Effects of aryl hydrocarbon receptor-mediated early life stage toxicity on lake trout populations in Lake Ontario during the 20th Century. Environ Sci Technol 37:3864-3877.

Couillard CM. 2002. A microscale test to measure petroleum oil toxicity to mummichog embryos. Environ Toxicol Chem 17:195-202.

Exxon Valdez Oil Spill Trustee Council. 2002. Annual status report 2002. Anchorage (AK): Exxon Valdez Oil Spill Trustee Council. $16 \mathrm{p}$

Forbes VE, Sibly RM, Calow R. 2001. Toxicant impacts on density-limited populations: A critical review of theory, practice, and results. Ecol App/ 11:1249-1257.

Fulton MH, Scott Gl, Fortner A, Bidleman TF, Ngabe B. 1993. The effects of urbanization on small high salinity estuaries of the southeastern United States. Arch Environ Contam Toxicol 25:476-484.

Geiger HJ, Bue BG, Sharr S, Wertheimer AC, Willette TM. 1996. A life-history approach to estimating damage to Prince William Sound pink salmon caused by the Exxon Valdez oil spill. Am Fish Soc Symp 18:487-498.

Grant A. 1998. Population consequences of chronic toxicity: Incorporating density dependence into the analysis of life table response experiments. Ecolog Model 105:325-335.

Halupka KC, Willson MF, Bryant MD, Everest FH. 1995. Biological characteristics and population status of anadromous salmon in Southeast Alaska. Juneau (AK): Forestry Sciences Laboratory, Pacific Northwest Research Station.

Hawkins SA, Billiard SM, Tabash SP, Brown RS, Hodson PV. 2002. Altering cytochrome P4501A activity affects aromatic hydrocarbon metabolism and toxicity in rainbow trout (Oncorhynchus mykiss). Environ Toxicol Chem 21:1845-1853

Heintz RA. 2001. Effects of oiled incubation substrate on pink salmon reproduction. Exxon Valdez oil spill restoration project annual report (restoration project 01476), Juneau (AK): US Department of Commerce, National Oceanic and Atmospheric Administration, National Marine Fisheries Service, Alaska Fisheries Science Center.

Heintz RA, Rice SD, Wertheimer AC, Bradshaw RF, Thrower FP, Joyce JE, Short JW. 2000. Delayed effects on growth and marine survival of pink salmon Oncorhynchus gorbuscha after exposure to crude oil during embryonic development. Mar Ecol Prog Ser 208:205-216.

Heintz RA, Short JW, Rice SD. 1999. Sensitivity of fish embryos to weathered crude oil: Part II. Increased mortality of pink salmon (Oncorhynchus gorbuscha) embryos incubating downstream from weathered Exxon Valdez crude oil. Environ Toxicol Chem 18:494-503.

Kennish MJ. 2002. Environmental threats and environmental future of estuaries. Environ Conserv 29:78-107.

Landahl JT, Johnson LL. 1993. Contaminant exposure and population growth of English sole in Puget Sound, Washington: The need for better early life-history data. Am Fish Soc Symp 14:117-124.

Leblanc JC, Couillard CM, Brethes JCF. 1997. Modifications of the reproductive period in mummichog (Fundulus heteroclitus) living downstream from a bleached kraft pulp mill in Miramichi Estuary, New Brunswick, Canada. Can J Fish Aquat Sci 54:2564-2573.

Levin LA, Caswell H, Bridges T, DiBacco C, Cabrera D, Plaia G. 1996. Demographic responses of estuarine polychaetes to pollutants: Life table response experiments. Ecol App/ 6:1295-1313.
Linke-Gamenick I, Forbes VE, Sibly RM. 1999. Density-dependent effects of a toxicant on life-history traits and population dynamics of a capitellid polychaete. Mar Ecol Prog Ser 184:139-148.

Marty GD, Short JW, Dambach DM, Willits JH, Heintz RA, Rice SD, Stegeman JJ, Hinton DE. 1997. Ascites, premature emergence, increased gonadal cell apoptosis, and cytochrome P4501A induction in pink salmon larvae continuously exposed to oil-contaminated gravel during development. Can J Zool 75:989-1007.

McNeil WJ. 1964. Red superimposition and egg capacity of pink salmon spawning beds. J Fish Res Board Can 21:1385-1396.

Menzie CA, Hoeppner SS, Cura JJ, Freshman JS, LaFrey EN. 2002. Urban and suburban storm water runoff as a source of polycyclic aromatic hydrocarbons (PAHs) to Massachusetts estuarine and coastal environments. Estuaries 25:165-176.

Meyer JN, Di Giulio RT. 2003. Heritable adaptation and fitness costs in killifish (Fundulus heteroclitus) inhabiting a polluted estuary. Ecol Appl 13:490-503.

Miller DH, Ankley GT. 2004. Modeling impacts on populations: Fathead minnow (Pimphales promelas) exposure to the endocrine disruptor $17 \beta$-trenbolone as a case study. Ecotoxicol Environ Saf 59:1-9.

National Research Council. 2002. Oil in the sea III inputs, fates, and effects. Washington DC: National Academies.

Nöel HL, Hopkins SP, Hutchinson TH, Williams TD, Sibly RM. 2006. Population growth rate and carrying capacity for springtails Folsomia candida exposed to ivermectin. Ecol Appl 16:656-665.

Ownby DR, Newman MC, Mulvey M, Vogelbein WK, Unger MA, Arzayus LF. 2002 Fish (Fundulus heteroclitus) populations with different exposure histories differ in tolerance of creosote-contaminated sediments. Environ Toxicol Chem 21:1897-1902

Pella JJ, Myren RT. 1974. Caveats concerning evaluation of effects of logging on salmon production in southeastern Alaska from biological information. Northw Sci 48:132-144.

Quinn TJ, Deriso RB. 1999. Quantitative fish dynamics. New York (NY): Oxford University Press. 542 p.

Roberts CM, Hawkins JP. 1999. Extinction risk in the sea. TREE 14:241-246.

Roy NK, Stabile J, Seeb JE, Habicht C, Wirgin I. 1999. High frequency of K-ras mutations in pink salmon embryos experimentally exposed to Exxon Valdez oil. Environ Toxicol Chem 18:1521-1528.

Schaaf WE, Peters DS, Coston-Clements L, Vaughan DS, Krouse CW. 1993. A simulation model of how life history strategies mediate pollution effects on fish populations. Estuaries 16:697-702.

Snell TW, Serra M. 2000. Using probability of extinction to evaluate the ecological significance of toxicant effects. Environ Toxicol Chem 19:2257-2263.

Spromberg JA, Meador JP. 2005. Relating results of chronic toxicity responses to population-level effects: Modeling effects on wild Chinook salmon populations. Integr Environ Assess Manag 1:9-21.

Steyermark AC, Spotila JR, Gillette D, Isseroff H. 1999. Biomarkers indicate health problems in brown bullheads from the industrialized Schuylkill River, Philadelphia. Trans Am Fish Soc 128:328-338.

Van Den Heuvel MR, Ellis RJ. 2002. Timing of exposure to a pulp and paper effluent influences the manifestation of reproductive effects in rainbow trout. Environ Toxicol Chem 21:2338-2347.

White PA, Robitaille S, Rasmussen JB. 1999. Heritable reproductive effects of benzo[a]pyrene on the fathead minnow (Pimphales promelas). Environ Toxicol Chem 18:1843-1847.

Zeng EY, Vista CL. 1997. Organic pollutants in the coastal environment off San Diego, California. 1. Source identification and assessment by compositional indices of polycyclic aromatic hydrocarbons. Environ Toxicol Chem 16:179188. 